\title{
Evaluation and Immunolocalization of BMP4 and FGF8 in Odontogenic Cyst and Tumors
}

\author{
Neeti Swarup $\mathbb{D}^{1},{ }^{1}$ Meghanand T. Nayak $\mathbb{D}^{2},{ }^{2}$ Zoya Chowdhary ${ }^{(D},{ }^{3}$ Monica Mehendiratta $\left(\mathbb{D},{ }^{4}\right.$ \\ Shikha Khatana $\left(1,{ }^{5}\right.$ Su Jung Choi $\odot{ }^{6},{ }^{6}$ and Chandarani Sagolsem $\left(\oplus^{7}\right.$ \\ ${ }^{1}$ Department of Oral Pathology and Microbiology, Daswani Dental College and Research Centre, Kota, India \\ ${ }^{2}$ Department of Oral Pathology and Microbiology, Teerthanker Mahaveer Dental College and Research Centre, Moradabad, India \\ ${ }^{3}$ Department of Periodontology, Indira Gandhi Government Dental College and Hospital, Jammu, India \\ ${ }^{4}$ Department of Oral Pathology and Microbiology, ITS Dental College, Greater Noida, India \\ ${ }^{5}$ Department of Oral Pathology and Microbiology, Sudha Rustagi College of Dental Sciences, Faridabad, India \\ ${ }^{6}$ Department of Oral Pathology, School of Dentistry, Seoul National University, Seoul, Republic of Korea \\ ${ }^{7}$ Department of Conservative Dentistry and Endodontics, Daswani Dental College and Research Centre, Kota, India
}

Correspondence should be addressed to Zoya Chowdhary; dr.zoya1988@gmail.com

Received 25 February 2018; Revised 5 June 2018; Accepted 19 June 2018; Published 12 July 2018

Academic Editor: Monica Cantile

Copyright (C) 2018 Neeti Swarup et al. This is an open access article distributed under the Creative Commons Attribution License, which permits unrestricted use, distribution, and reproduction in any medium, provided the original work is properly cited.

\begin{abstract}
Growth factors like bone morphogenetic protein 4 (BMP4) and fibroblast growth factor 8 (FGF8) play a major role in organogenesis and specifically in odontogenesis. They are also believed to have a role in oncogenesis. Thus, any discrepancies in their standard behavior and activity would lead to serious abnormalities including odontogenic cyst and tumors. The present research work investigated the expression of BMP4 and FGF8 in odontogenic tumors (OT) and cyst as well as developing tooth germs to elucidate their roles. Dental organs of various odontogenic stages and 30 OTs including solid multicystic ameloblastomas (SMA, 10 cases), ameloblastic fibroma (AF, 10 cases), odontogenic myxoma (OM, 10 cases), and odontogenic cysts: odontogenic keratocyst (OKC, 10 cases) were evaluated in both epithelial and mesenchymal components for the expression of BMP4 and FGF8 using immunohistochemistry. The epithelial nuclear expression of BMP4 was highest in OKC (9 cases) while FGF8 was highest in SMA (10 cases). The mesenchymal nuclear expression of both BMP4 (8 cases) ( $p=0.001)$ and FGF8 ( 9 cases) $(p=0.045)$ were significantly high in OMs among all OTs. Both growth factors were actively expressed in different stages of tooth development. The expression of BMP4 and FGF8 corelates well with the proliferative component of the pathologies, indicating a possible role in the pathogenesis and progression.
\end{abstract}

\section{Introduction}

Odontogenic tumors (OTs) are a unique group of neoplasms, derived from tooth forming apparatus or its remnants, hence found exclusively in the jaws or associated soft tissue [1]. In 2005, World Health Organization (WHO) classified them broadly under benign and malignant and subclassified them further based on tissue of origin, that is, epithelial, ectomesenchymal, or both; this however led to inclusion and classification of odontogenic keratocyst (OKC) as keratocystic odontogenic tumor in group 1 tumors [2].

Recently, the status OKC was addressed and classified into the category of cyst. Based on WHO histological classification of OTs and odontogenic cysts (2017), three benign tumors were considered for the study, namely, solid multicystic ameloblastoma (SMA) from group1 (epithelial origin); ameloblastic fibroma (AF) from group 2 (mixed origin); and odontogenic myxoma (OM) from group 3 (ectomesenchymal origin) and OKC was included in the study owing to its aggressive nature [3].

The pathogenesis of OTs like any tumor is dependent on two factors, tumor initiating factors and tumor progression factors. Genes expressing these factors in OTs show a striking resemblance to those expressed during odontogenesis [1]. OKC arises from remnants of the dental lamina (rest of Serres) due to inactivation of PTCH1, which activates Shh 
pathway leading to excessive proliferation [3]. Odontogenesis is a very complex process monitored by intricate interactions between homeobox genes and their signaling molecules; sonic hedgehog (Shh), Wnt, BMP, and FGF [4].

Bone morphogenetic proteins (BMP) is a member of transforming growth factor (TGF $\beta$ ) superfamily. There are more than 30 known types of BMP and are well known as the signaling molecule, which plays a crucial role in cellular proliferation, extracellular matrix production along with differentiation of neoplastic tissues [5]. BMP4 plays a key role in Msx-1-dependent mesenchymal odontogenic signaling for tooth morphogenesis, and it is also linked to epidermal differentiation $[6,7]$.

Fibroblast growth factors (FGF) perform a variety of cellular processes such as stemness, proliferation, and antiapoptosis. There are 18 members which are grouped under 6 subfamilies along with four receptors [8]. FGF8 plays an important role during embryogenesis and is required during the initiation of odontogenesis [9].

Odontogenic pathologies present with a wide array of molecular variations. The two described factors have been scarcely studied in OTs and OKC. The study of these two factors can lead to a better understanding of the behavior of OTs and OKC and pave the way for establishing better pathological evaluation processes and development of novel therapies. On this premise, we conducted the study to evaluate the expression of these two growth factors in OTs and OKC and furthermore compared with that of different stages of odontogenesis.

\section{Materials and Methods}

2.1. Specimen Collection. Forty cases of odontogenic pathologies, 10 cases for each SMA, OKC, AF, and OM, were retrieved from archives of six different institutes of North India: Teerthanker Mahaveer Dental College \& Research Centre, Moradabad $(n=15)$; Institute of Dental Sciences, Bareilly $(n=12)$; Sudha Rustagi Dental College and Research Centre, Faridabad $(n=5)$; Subharti Dental College, Meerut $(n=4)$; Surendra Dental College \& Research Centre, Sri Ganganagar $(n=2)$; and MedAid India, Noida $(n=2)$. Sections from mandibular tissue of chemically aborted fetus were obtained from Teerthanker Mahaveer Medical College and Research Centre to procure stages of odontogenesis: dental lamina, bud stage, cap stage, and bell stage. Three sections each of 3 microns thickness were obtained from formalin fixed paraffin embedded tissue. Hematoxylin and eosin stained section was used for diagnosis using WHO criteria (2017). Two sections were obtained on poly-L-lysine-coated slides and stained with BMP4 and FGF8 using immunohistochemical methods. Few additional sections of cases of epidermoid carcinoma and ductal carcinoma of breast were included to serve as controls for BMP4 and FGF8, respectively. The study was approved by the Institutional Ethical Committee, Teerthanker Mahaveer Dental College and Research Centre, Teerthanker Mahaveer University, Moradabad, U.P., India.
2.2. Immunohistochemistry. The deparaffinised sections on poly-L-lysine-coated slides after rehydration were subjected to the immunohistochemical procedure.

2.3. BMP4. The antibody clone used for detection of BMP4 was GTX100875 (Genetex Hsinchu City, Taiwan, polyclonal antibody). The antibody was diluted to a dilution of $1: 100$ by adding $1 \mu \mathrm{l}$ antibody to $99 \mu \mathrm{l}$ of Renoir Red Diluent ${ }^{\circledR}$. Antigen retrieval was done by boiling the sections obtained on poly-L-ysine slides in Diva Decloaker antigen retrieval solution, BioCare DV2005 L2J (low pH 6.5), which was obtained by mixing $1 \mathrm{ml}$ Diva Decloaker to $19 \mathrm{ml}$ of distilled water in a pressure cooker at $120^{\circ} \mathrm{C}$ for $20-25 \mathrm{~min}$. The sections were then incubated with antibody at room temperature in a humidity chamber for 1 hour and 15 minutes. For antibody detection, Leica Novolink ${ }^{\mathrm{TM}}$ Novocastra Polymer HRP Kit \#RE 7290-CE was used as per directions. Epidermoid carcinoma was used as a positive control, and the negative control staining was performed without primary antibody.

2.4. FGF8. The antibody clone used for detection of FGF8 was PA1216 (Boster, Pleasanton, CA, polyclonal antibody). The lyophilised antibody was reconstituted by adding $0.2 \mathrm{ml}$ distilled water to the $100 \mu \mathrm{g}$ antibody which yielded $500 \mu \mathrm{g} / \mathrm{ml}$. The reconstituted antibody was diluted to a dilution of $1: 200$ to yield a concentration of $0.5 \mu \mathrm{g} / \mathrm{ml}$ in Renoir Red Diluent. Antigen retrieval was done by boiling the sections obtained on poly-L-lysine slides in Diva Decloaker antigen retrieval solution, BioCare DV2005 L2J (low pH 6.5), which was obtained by mixing $1 \mathrm{ml}$ Diva Decloaker to $19 \mathrm{ml}$ of distilled water in a pressure cooker at $120^{\circ} \mathrm{C}$ for $20-25 \mathrm{~min}$. The sections were then incubated with antibody at room temperature in a humidity chamber for 1 hour and 15 minutes. For antibody detection, Leica Novolink Novocastra Polymer HRP Kit \#RE 7290-CE was used as per directions. Ductal carcinoma of the breast was used as a positive control, and the negative control staining was performed without primary antibody.

The stained specimens were then sequentially examined by two observers under $4 \mathrm{x}, 10 \mathrm{x}$ and $40 \mathrm{x}$ magnification for BMP4 and FGF8 expression in the epithelial and mesenchymal component and were assessed for cytoplasmic or nuclear reactivity. A total of five high power fields were observed. The specimens were scored on the basis of the number of cells stained as described below.

Scoring criteria:

(1) -/negative: no cells were stained.

(2) + /positive: $<20$ percent cells were stained.

(3) $++/$ strong positive: $20-80$ percent cells were stained.

(4) $+++/$ very strong positive: $>80$ percent cells were stained.

2.5. Statistical Evaluation. The results obtained were subjected to statistical evaluation using SPSS version 21.0. Chisquare test was applied for statistical evaluation for intergroup comparison between OKC and OTs but due to smaller 


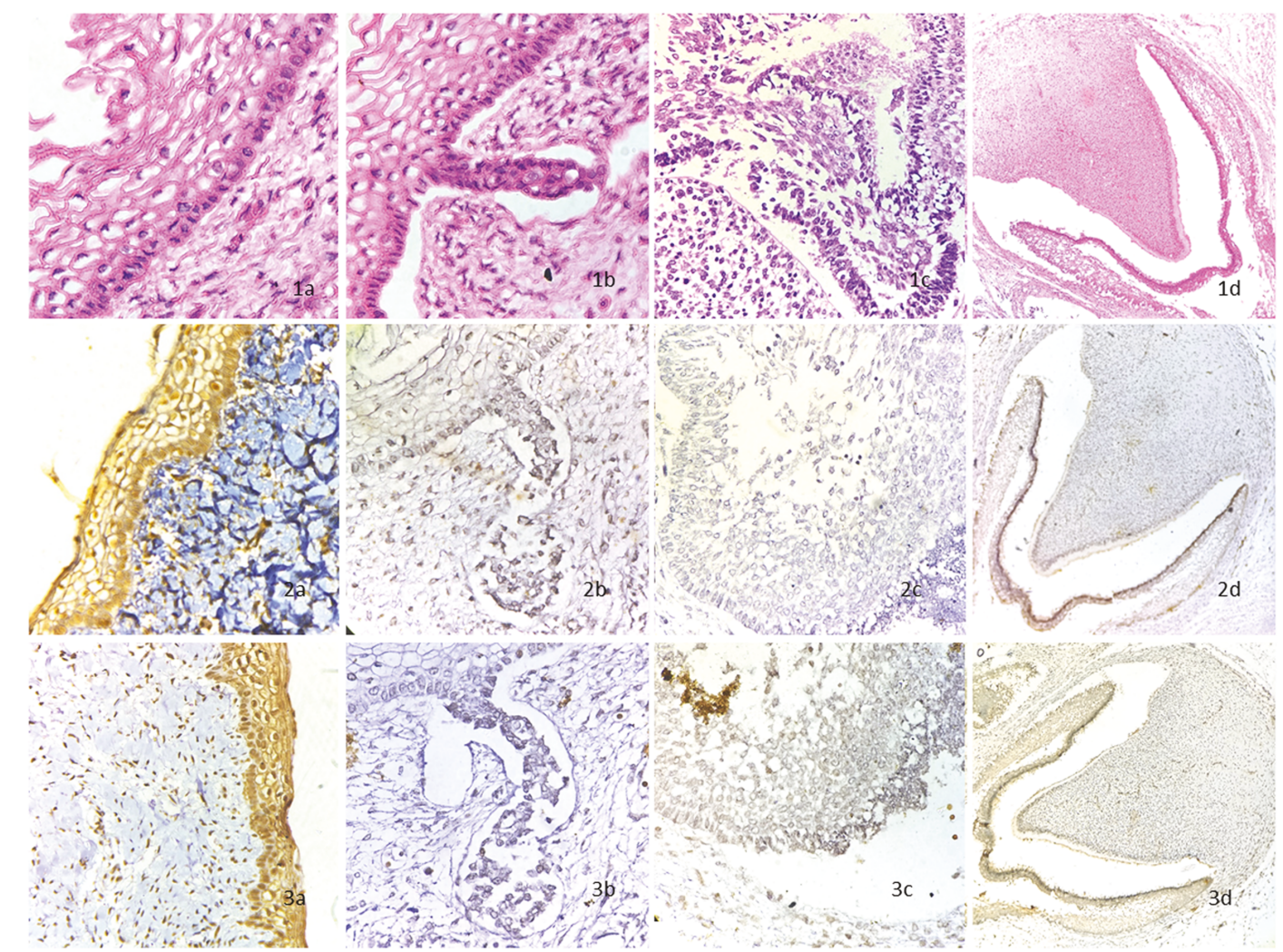

FIGURE 1: Staining of stages of odontogenesis: (1a) shows $\mathrm{H}$ and $\mathrm{E}$ stained dental lamina at $\mathrm{x} 400$; (1b) shows $\mathrm{H}$ and $\mathrm{E}$ stained bud stage at $\mathrm{x} 400$; (1c) shows $\mathrm{H}$ and E stained cap stage at $\mathrm{x} 400$; (1d) shows $\mathrm{H}$ and $\mathrm{E}$ stained bell stage at $\mathrm{x} 100$. (2a) shows BMP4 stained dental lamina at $\mathrm{x} 400$, the epithelium is strongly positive with moderate reactivity in ectomesenchyme; (2b) shows BMP4 stained bud stage at $\mathrm{x} 400$, the epithelium is nonreactive with slight reactivity in ectomesenchyme; (2c) shows BMP4 stained cap stage at x400, the epithelium and ectomesemchyme are nonreactive; (2d) shows BMP4 stained bell stage at x100, the epithelium and dental papilla is strongly positive. (3a) shows FGF8 stained dental lamina at $\mathrm{x} 400$, the epithelium is strongly positive with moderate reactivity in ectomesenchyme; (3b) shows FGF8 stained bud stage at x400, the epithelium and ectomesenchyme are nonreactive; (3c) shows FGF8 stained cap stage at x400, the epithelium and ectomesenchyme are nonreactive; (3d) shows FGF8 stained bell stage at x100, the epithelium and dental papilla is strongly positive.

sample size for intragroup comparison between growth factors percentage was calculated for evaluation of data.

\section{Results}

The stained specimens (Figures 1 and 2) were scored according to the criteria. The results obtained are summarized in Tables 1, 2, and 3.

3.1. Stages of Odontogenesis. Dental lamina demonstrated very strong cytoplasmic positivity for BMP4 and FGF8 in the ectodermal and ectomesenchymal components.

Bud stage dental organ demonstrated slight nuclear reactivity for BMP4 in the dental epithelium and weaker staining in the stroma. Reactivity for FGF8 was only found in the dental epithelium in both nucleus and cytoplasm.

The odontogenic epithelial components and ectomesenchymal components during the cap stage were reactive for FGF8 with mild nuclear and cytoplasmic positivity.

The bell stage dental organ showed a stronger cytoplasmic reactivity for both of these two factors in the epithelial and ectomesenchymal component.
3.2. Ameloblastoma. The epithelial component of all 10 out of 10 cases $(100 \%)$ of SMA demonstrated nuclear localization of FGF8 but only 7 out of 10 cases (70\%) were reactive to BMP4, whereas the mesenchyme was less reactive and demonstrated a similar result for both growth factors.

3.3. OKC. The nuclear localization of BMP4 was found in the epithelial component of 9 out of 10 cases (90\%) of OKC whereas only 7 out of 10 cases $(70 \%)$ demonstrated nuclear localization of FGF8, while the mesenchymal component was slightly more reactive for BMP4, 8 out of 10 cases (80\%), as compared to FGF8, 6 out of 10 cases (60\%).

3.4. Ameloblastic Fibroma. AFs showed a comparable nuclear and cytoplasmic expression in the epithelial component for BMP4, 7 out of 10 cases (70\%), and FGF8, 6 cases out of 10 (60\%). The mesenchymal component was slightly more reactive for BMP4, 6 out of 10 cases (60\%).

3.5. Odontogenic Myxoma. The nuclear expression of FGF8, 9 out of 10 cases (90\%), was stronger as compared to BMP4, 8 out of 10 cases $(80 \%)$, whereas the cytoplasmic reactivity was comparable for both the growth factors. 


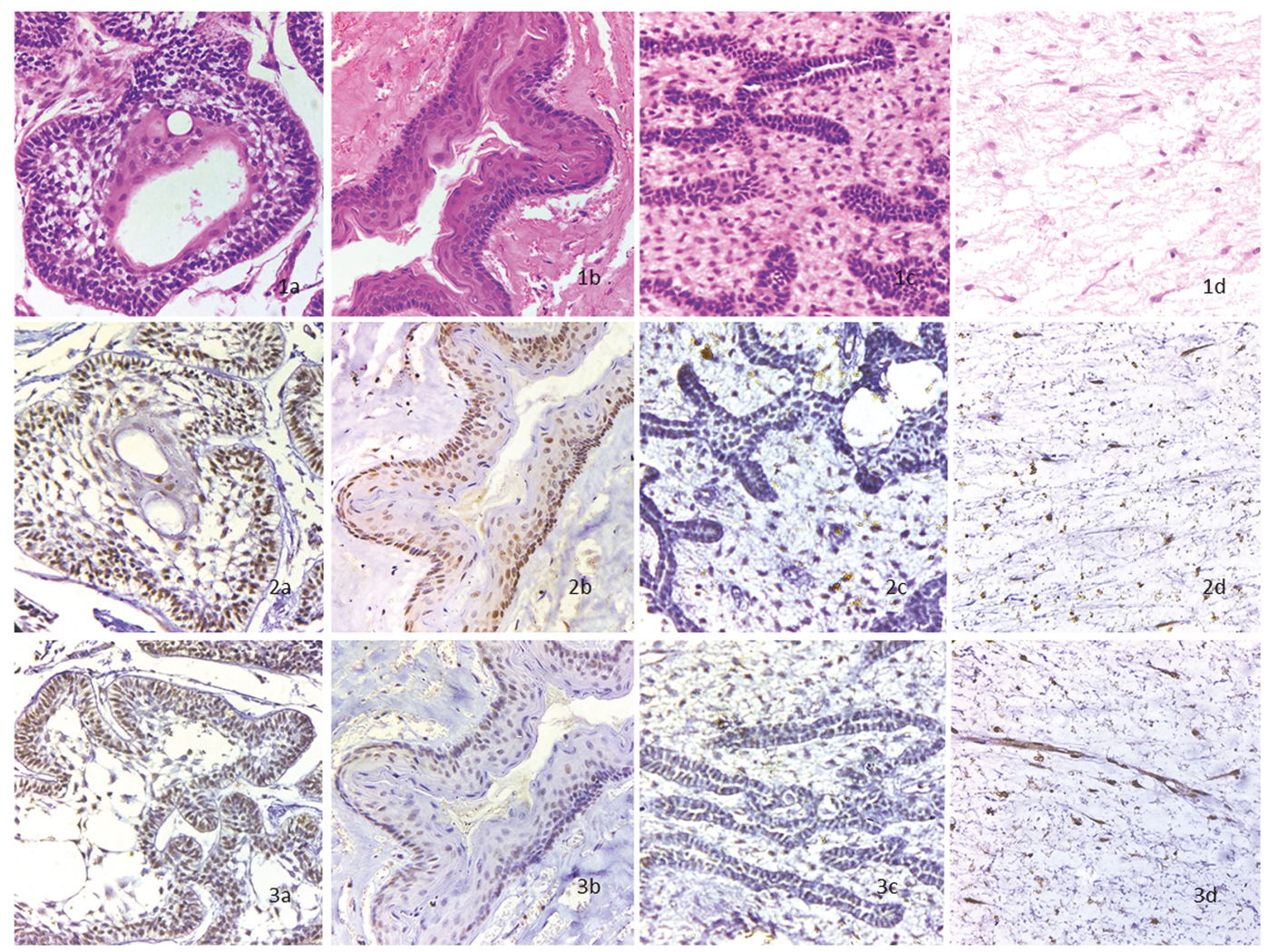

FIGURE 2: Staining of odontogenic tumors and odontogenic cyst: (1a) shows H and E stained SMA at x400; (1b) shows H and E stained OKC at $\mathrm{x} 400$; (1c) shows $\mathrm{H}$ and $\mathrm{E}$ stained AF at $\mathrm{x} 400$; (1d) shows $\mathrm{H}$ and $\mathrm{E}$ stained OM at $\mathrm{x} 400$. (2a) shows BMP4 stained SMA at $\mathrm{x} 400$, the epithelium is positive with slight reactivity in mesenchyme; (2b) shows BMP4 stained OKC at $\mathrm{x} 400$, the epithelium is strongly reactive with moderate reactivity in mesenchyme; (2c) shows BMP4 stained AF at x400, the epithelium and mesenchyme are mildly reactive; (2d) shows BMP4 stained OM at $\mathrm{x} 400$, the mesenchymal component is strongly positive. (3a) shows FGF8 stained SMA at $\mathrm{x} 400$, the epithelium is strongly positive with moderate reactivity in mesenchyme; (3b) shows FGF8 stained OKC at x400, the epithelium is positive and mesenchyme shows slight reactivity; (3c) shows FGF8 stained AF at x400, the epithelium and mesenchyme are mildly reactive; (3d) shows FGF8 stained OM at $\mathrm{x} 400$, the mesenchyme is strongly positive.

3.6. BMP4 in OTs and OKC. The nuclear localization in the epithelial component was found to be highest in OKC (9 out of 10 cases, 90\%), while the cytoplasmic reactivity was higher in AFs (5 out of 10 cases, 50\%). The mesenchymal component of OMs (8 out of 10 cases, $80 \%$ ) had highest nuclear reactivity for BMP4 $(p=0.001)$ while OKC (7 out of 10 cases, $70 \%$ ) had greater cytoplasmic reactivity.

3.7. FGF8 in OTs and OKC. SMA (10 out of 10 cases, 100\%) had the highest nuclear reactivity in the epithelial component for the same, while the cytoplasmic reactivity was higher in AFs (4 out of 10 cases, $40 \%$ ). The mesenchymal component of OMs ( 9 out of 10 cases, $90 \%$ ) had the highest nuclear reactivity for FGF8 $(p=0.045)$, while OKC ( 4 out of 10 cases, $40 \%$ ) had greater cytoplasmic reactivity.

\section{Discussion}

Various components of the odontogenic apparatus can be identified as the source of origin for OTs and OKC. They may comprise of the odontogenic epithelium, the ectomesenchyme or may include both the components. The pathogenesis of OTs and OKC is a complex process, which at some stages resembles the odontogenic process. Odontogenesis is a highly regulated process monitored by regulatory genes and signaling factors. The regulatory genes belong to the homeobox family, and signaling factors belong to the BMP, FGF, Wnt, and Shh families [4].

BMPs are chief regulators of development, playing a major role in the organogenetic process, regenerative processes, and carcinogenetic process $[5,10]$. By regulating target gene transcription, various cellular processes are controlled by these growth factors, such as proliferation, differentiation, apoptosis, migration, and angiogenesis. BMPs play a dual role in cancer cell regulation; they upregulate growth in certain tumors and downregulate growth in other tumors [10].

BMP4 is a member of BMP family and transforming growth factor beta-1 (TGF $\beta 1$ ) superfamily of secretory signaling molecules that play vital roles in embryonic development [11]. It is a critical regulatory molecule performing a wide array of functions during development, including mesoderm induction, limb formation, tooth development, bone induction, and fracture repair.

BMP4 contributes to various cancer-associated phenotypes, including cell growth, differentiation, migration, invasion, and angiogenesis, which is critical for cancer 
TABLE 1: Evaluation and immunohistochemical score of stages of odontogenesis.

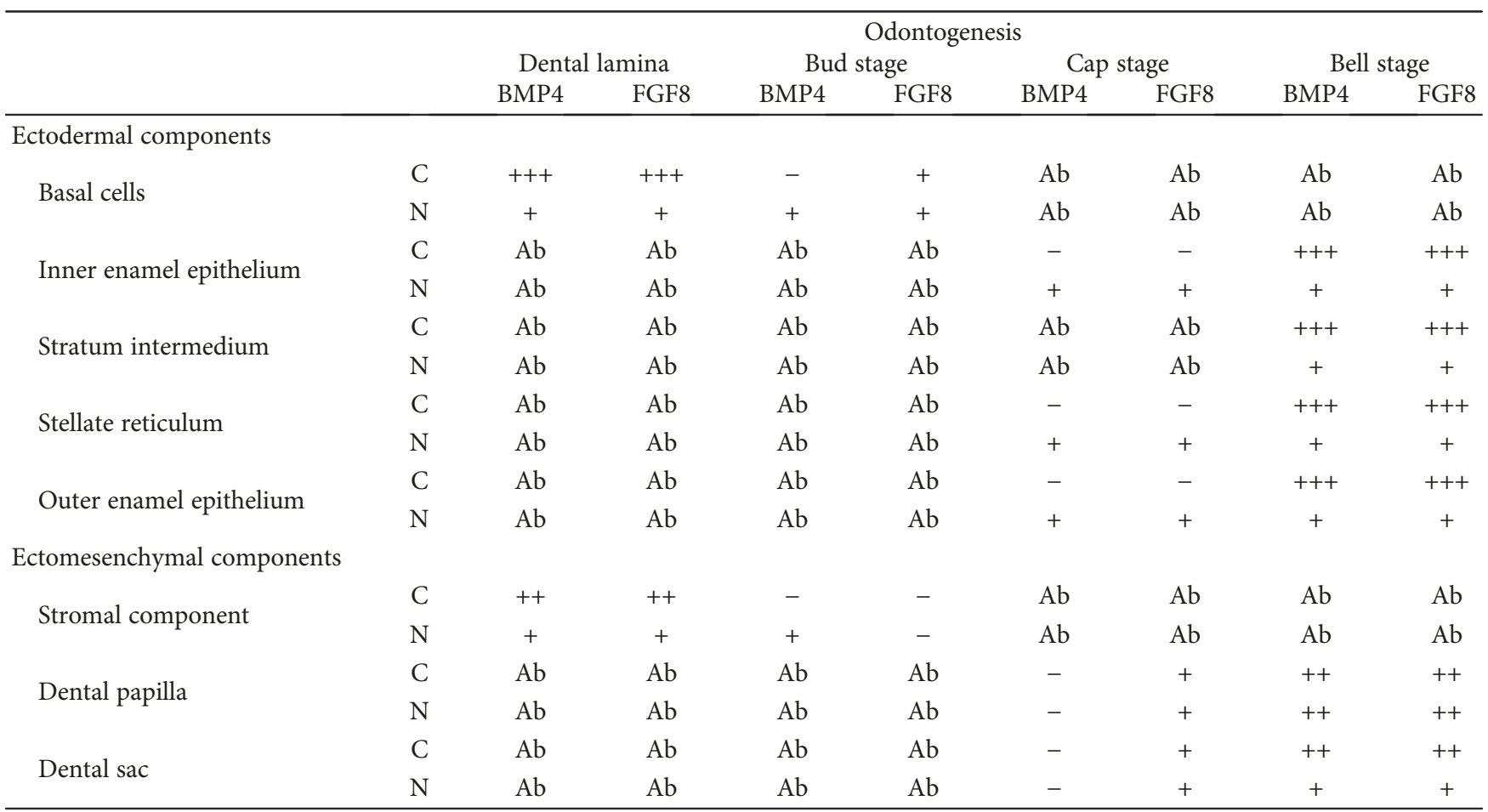

C: cytoplasm; N: nucleus; Ab: absent.

development and progression. It has a dual role as a proapoptotic and antiapoptotic factor in different tumors. BMP4 is also an important regulator of cell migration and invasion and is known to induce the epithelial mesenchymal transition (EMT), which grants mobility to cancer cells and eventually aid in metastasis [12]. BMP FGF axis maintains proliferation of basal keratinocytes and plays a major role in epidermal stratification [7].

The family of FGFs regulates an ample number of developmental processes, including branching morphogenesis, brain patterning, and limb development $[13,14]$. FGFs and their receptors control a wide range of biological functions, regulating cellular proliferation, survival, migration, and differentiation. Compagni et al. [15] indicated that FGFs play a crucial role in tumor angiogenesis. FGF8 plays a pivotal role during development and also during carcinogenesis.

FGF8 was originally referred to as androgen-induced growth factor (AIGF) as it played a role in the growth of androgen-dependent growth of mouse mammary tumor cell line [16]. FGF8 was found to impart proliferative and metastatic capacity of colorectal cancer cells [17]. It is also responsible for maintaining the progenitor status and fate determination of cranial neural crest cells [18]. DaphnaIken et al. [19] demonstrated that production of FGF8b, and other FGF8 isoforms, contributed to oncogenesis in mammary and salivary glands, and that ovarian expression results in stromal hyperplasia.

In the present study, SMA showed stronger expression of FGF8 than BMP4 expression. FGF8 was found to induce odontogenic epithelium during the early stages of odontogenesis [9]. Zhong et al. [20] found that FGF8 overexpression in prostate epithelium led to prostate intraepithelial neoplasia. We may conclude that increased expression of FGF8 might play a role in tumor initiation and progression by inducing the odontogenic epithelium in cases of SMA. Expression of BMP4 in SMA was found to be in accordance with the study conducted by Kumamoto and Ooya [21]. There was a decreased expression of BMP4 in granular cell SMA in a study conducted by Sathi et al. [22] in 2007, but no cases of granular cell SMA were included in our study. Expression of BMP4 in cells with squamous metaplasia in the acanthomatous type of SMA was a distinctive finding.

In the present study, the BMP4 expression was stronger than FGF8 in cases of OKC. Increased expression of BMP4 in OKC was found to be in accordance with the study conducted by Kim et al. [23], but they were contrary to the results by Gao et al. [24], which indirectly concluded the downregulation of BMP4 signaling due to decreased expression of Smad4. BMP4 was found to be responsible for proliferative abilities of basal cell keratinocytes [7]. The study conducted by Heikinheimo et al. [25] suggests that SMA displayed an odontogenic fate while OKC displayed squamous epithelial fate. The preferential expression of FGF8 for SMA and BMP4 in OKC in our study may well explain the odontogenic and squamous epithelial fates, respectively.

The results obtained in our study suggest that the expression of BMP4 and FGF8 were equivalent in AF with slightly more cytoplasmic reactivity, which resembled the expression of BMP4 and FGF8 in the bell stage of odontogenesis. The milder expression and resemblance to the mature stage of tooth development may explain the indolent behavior of this tumor and more differentiated/mature neoplastic cells. 


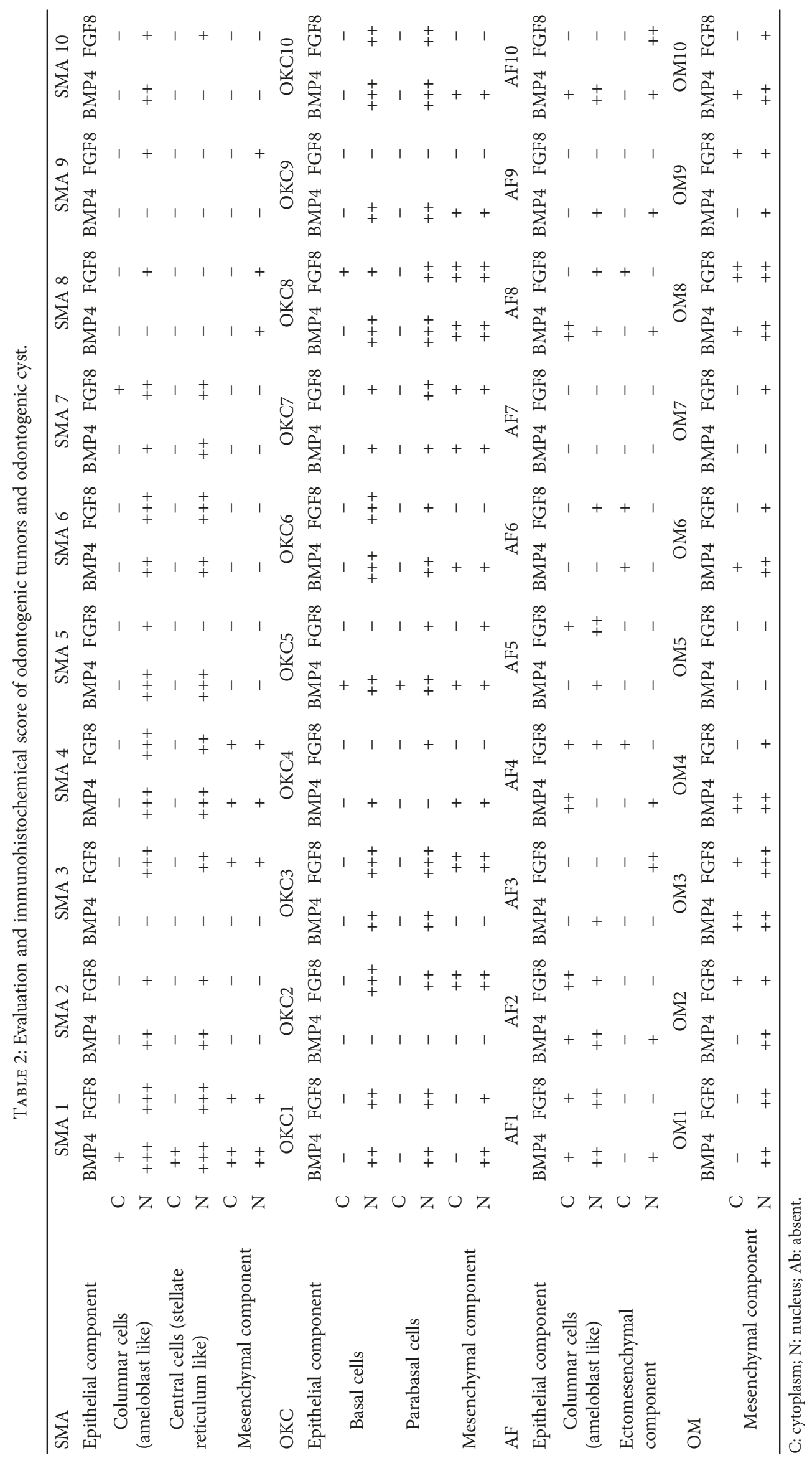


TABLE 3: Intergroup comparison of expression of BMP4 and FGF8 in odontogenic tumors and odontogenic cyst.

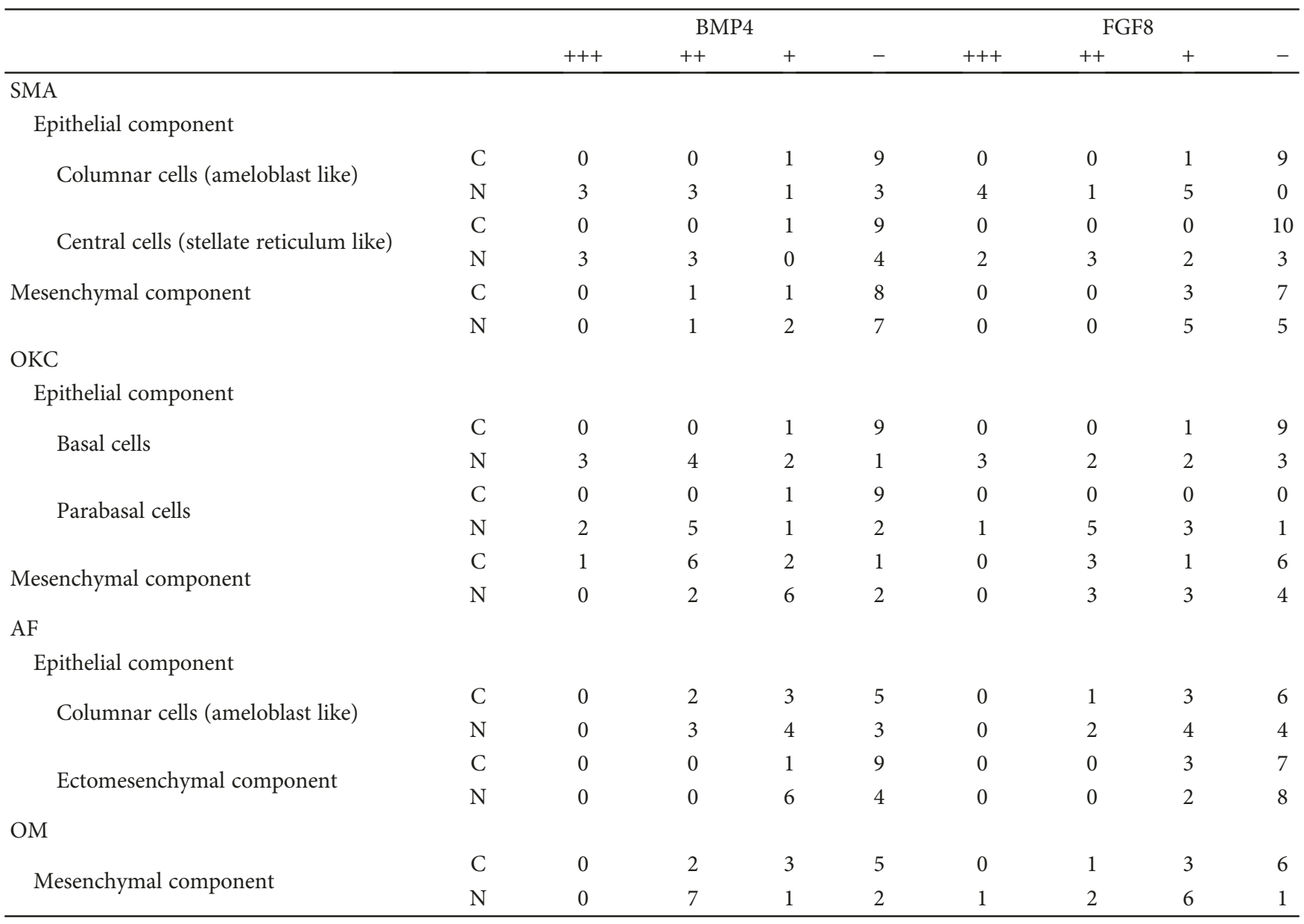

C: cytoplasm; N: nucleus.

Similar conclusions were drawn from the results obtained by So et al. [26], who revealed a comparable expression of FGF2 in $\mathrm{AF}$ and ameloblastic fibroodontoma and histodifferentiation stage of odontogenesis.

The expression of FGF8 and BMP4 were comparable with slightly more reactivity for FGF8 in OMs. Immunohistochemical studies for OMs regarding the two or related growth factors have been very scarce. Based on results of the study conducted by Molina et al. [27], expression of VEGF-A and ORM-1 may be associated with angiogenesis and tumor structural viscosity which may influence tumor growth in OMs. However, the limited data on upregulation of expression of FGF8 in English literature search was found in myxoinflammatory fibroblastic sarcoma [28] and few other tumors [14, 17, 19]. FGF8 was also found to have a synergistic role with VEGF in prostate cancer [29]. The results indicate a possible relation between FGF8 and initiation, progression and its interaction with other factors in the pathogenesis of the OMs, which requires further substantiation. We may conclude that an increased expression of BMP4 and FGF8 in OM may be responsible for imparting it an aggressive nature.

Overall, the reactivity for BMP4 was seen to be the highest in OKC when considering both epithelial and mesenchymal component among all tumors, but the mesenchymal reactivity was highest in OM. The epithelial component of all SMA showed reactivity for FGF8, whereas the mesenchymal reactivity was highest in OMs. Among all tumors, the overall reactivity for FGF8 in AF was low when compared to other tumors, although AF had a significant epithelial positivity for cytoplasmic BMP4.

Our results for expression of BMP4 in SMA were contradictory to Gao et al. [30]; they concluded from their study that SMA was nonreactive for BMP McAb but positive for group 2 and group 3 OTs. They attributed this expression to its role in hard tissue formation; however, newer roles of BMP4 in tumorigenesis and development have been elucidated by other researchers and now the function of BMP4 is no longer confined to hard tissue formation [30]. The expression of BMP4 and FGF8 in SMA and OKC noted in the present study were in accordance with the study conducted by Pimentel [31] in 2015.

Both BMP4 and FGF8 were found to be positive in the epithelial and mesenchymal components of different tumors under varying degrees, suggesting that they are involved in epithelial and mesenchymal interactions via paracrine and autocrine mechanisms [12]. 
Studies demonstrate that BMP4 suppresses cell growth both in vitro and in vivo, and at the same time is able to induce migration, invasion, and EMT. The dual role makes it an interesting growth factor, which may explain the aggressive nature of tumors like OM and OKC [10]. Furthermore, expression of BMP4 by keratinizing components in OKC and acanthomatous SMA may indicate their role in squamous differentiation.

Overexpression of FGFs in the epithelium induces carcinogenesis through an autocrine signal loop, which may clarify the oncogenetic changes taking place in the odontogenic epithelial component leading to the pathogenesis of SMA.

The decreased reactivity of both factors in the case of AF, when compared to other odontogenic pathologies, may explain the indolent behavior of the tumor when compared to different tumors.

In conclusion, the results suggest that BMP4 and FGF8 are expressed in different odontogenic cyst and tumors with varying intensity based on their expected behavior. The greater expression for growth factors in aggressive pathologies may suggest an analogy to proliferative stages of odontogenesis. While, in pathologies with milder clinical course, a lesser expression for growth factors was observed which may be considered to parallel with different stages of odontogenesis. The results also indicate that BMP4 and FGF8 may play important roles in the pathogenesis of the odontogenic pathologies and furthermore regulate their clinical behavior, which requires further genetic and proteomic studies with larger sample sizes to better understand the participation of these elements in these pathologies.

\section{Data Availability}

The data used to support the findings of this study are available from the corresponding author upon request.

\section{Disclosure}

The current research was presented as a poster titled "Comparative Evaluation of BMP4 and FGF8 in Odontogenic Tumors" at the 18th International Congress on Oral Pathology and Medicine-XXV Conference of Indian Association of Oral and Maxillofacial Pathologists Conference, September 2016, Chennai, India. It was awarded Second Prize for Scientific Content and Quality of Presentation.

\section{Conflicts of Interest}

The authors state no conflicts of interest and alone are responsible for the content and writing of the paper.

\section{References}

[1] H. Kumamoto, "Molecular pathology of odontogenic tumors," Journal of Oral Pathology \& Medicine, vol. 35, no. 2, pp. 65-74, 2006.

[2] L. Barnes, J. W. Eveson, P. Reichart, and D. Sidransky, World Health Organization Classification of Tumours, Pathology and Genetics of Tumours of the Head and Neck, IARC Press, Lyon, 2005.
[3] A. K. El-Nagger, J. K. C. Chan, J. R. Grandis, T. Takata, and P. J. Slootweg, WHO Classification of Head and Neck Tumours, IARC Press, Lyon, 2017.

[4] I. Thesleff and T. Åberg, "Molecular regulation of tooth development," Bone, vol. 25, no. 1, pp. 123-125, 1999.

[5] P. Ducy and G. Karsenty, "The family of bone morphogenetic proteins," Kidney International, vol. 57, no. 6, pp. 2207-2214, 2000.

[6] M. Bei, K. Kratochwil, and R. L. Maas, "BMP4 rescues a noncell-autonomous function of Msx1 in tooth development," Development, vol. 127, no. 21, pp. 4711-4718, 2000.

[7] X.-J. Zhu, Y. Liu, Z.-M. Dai et al., "BMP-FGF signaling axis mediates Wnt-induced epidermal stratification in developing mammalian skin," PLoS Genetics, vol. 10, no. 10, article e1004687, 2014.

[8] C. M. Teven, E. M. Farina, J. Rivas, and R. R. Reid, "Fibroblast growth factor (FGF) signaling in development and skeletal diseases," Genes \& Diseases, vol. 1, no. 2, pp. 199-213, 2014.

[9] P. Kettunen, I. Karavanova, and I. Thesleff, "Responsiveness of developing dental tissues to fibroblast growth factors: expression of splicing alternatives of FGFR1, $-2,-3$, and of FGFR4; and stimulation of cell proliferation by FGF-2, $-4,-8$, and -9,” Developmental Genetics, vol. 22, no. 4, pp. 374-385, 1998.

[10] E. L. Alarmo and A. Kallioniemi, "Bone morphogenetic proteins in breast cancer: dual role in tumourigenesis?," Endocrine-Related Cancer, vol. 17, no. 2, pp. R123-R139, 2010.

[11] “Online Mendelian Inheritance in Man," OMIM ${ }^{\circledR}$.Johns Hopkins University, Baltimore, MD, USA, 2016, http://omim.org/ 112262.

[12] A. Kallioniemi, "Bone morphogenetic protein 4-a fascinating regulator of cancer cell behavior," Cancer Genetics, vol. 205, no. 6, pp. 267-277, 2012.

[13] A. Beenken and M. Mohammadi, "The FGF family: biology, pathophysiology and therapy," Nature Reviews Drug Discovery, vol. 8, no. 3, pp. 235-253, 2009.

[14] N. Turner and R. Grose, "Fibroblast growth factor signalling: from development to cancer," Nature Reviews Cancer, vol. 10, no. 2, pp. 116-129, 2010.

[15] A. Compagni, P. Wilgenbus, M. A. Impagnatiello, M. Cotten, and G. Christofori, "Fibroblast growth factors are required for efficient tumor angiogenesis," Cancer Research, vol. 60, no. 24, pp. 7163-7169, 2000.

[16] “Online Mendelian Inheritance in Man," OMIM ${ }^{\circledR}$.Johns Hopkins University, Baltimore, MD, USA, 2016, http://omim.org/ 600483.

[17] R. Liu, S. Huang, Y. Lei et al., "FGF8 promotes colorectal cancer growth and metastasis by activating YAP1," Oncotarget, vol. 6, no. 2, pp. 935-952, 2015.

[18] M. Shao, C. Liu, Y. Song et al., "FGF8 signaling sustains progenitor status and multipotency of cranial neural crestderived mesenchymal cells in vivo and in vitro," J Mol Cell Biol, vol. 7, no. 5, pp. 441-454, 2015.

[19] D. Daphna-Iken, D. B. Shankar, A. Lawshé, D. M. Ornitz, G. M. Shackleford, and C. A. MacArthur, "MMTV-Fgf8 transgenic mice develop mammary and salivary gland neoplasia and ovarian stromal hyperplasia," Oncogene, vol. 17, no. 21, pp. 2711-2717, 1998.

[20] C. Zhong, G. Saribekyan, C. P. Liao, M. B. Cohen, and P. RoyBurman, "Cooperation between FGF8b overexpression and PTEN deficiency in prostate tumorigenesis," Cancer Research, vol. 66, no. 4, pp. 2188-2194, 2006. 
[21] H. Kumamoto and K. Ooya, "Expression of bone morphogenetic proteins and their associated molecules in ameloblastomas and adenomatoid odontogenic tumors," Oral Diseases, vol. 12, no. 2, pp. 163-170, 2006.

[22] G. S. A. Sathi, P. P. Han, R. Tamamura et al., "Immunolocalization of cell signaling molecules in the granular cell ameloblastoma," Journal of Oral Pathology \& Medicine, vol. 36, no. 10, pp. 609-614, 2007.

[23] S.-G. Kim, B. E. Yang, S. H. Oh, S. K. Min, S. P. Hong, and J. Y. Choi, "The differential expression pattern of BMP-4 between the dentigerous cyst and the odontogenic keratocyst," Journal of Oral Pathology \& Medicine, vol. 34, no. 3, pp. 178-183, 2005.

[24] Y. Gao, G. Yang, T. Weng et al., "Disruption of Smad4 in odontoblasts causes multiple keratocystic odontogenic tumors and tooth malformation in mice," Molecular and Cellular Biology, vol. 29, no. 21, pp. 5941-5951, 2009.

[25] K. Heikinheimo, K. J. Kurppa, A. Laiho et al., "Early dental epithelial transcription factors distinguish ameloblastoma from keratocystic odontogenic tumor," Journal of Dental Research, vol. 94, no. 1, pp. 101-111, 2014.

[26] F. So, T. D. Daley, L. Jackson, and G. P. Wysocki, "Immunohistochemical localization of fibroblast growth factors FGF-1 and FGF-2, and receptors FGFR2 and FGFR3 in the epithelium of human odontogenic cysts and tumors," Journal of Oral Pathology \& Medicine, vol. 30, no. 7, pp. 428-433, 2001.

[27] R. B. Molina, A. M. Taylor, H. D. Malagón et al., "Immunolocalization of VEGF-A and orosomucoid-1 in odontogenic myxoma," Romanian Journal of Morphology and Embryology, vol. 56, pp. 465-473, 2015.

[28] K. H. Hallor, R. Sciot, J. Staaf et al., "Two genetic pathways, $\mathrm{t}(1 ; 10)$ and amplification of 3p11-12, in myxoinflammatory fibroblastic sarcoma, haemosiderotic fibrolipomatous tumour, and morphologically similar lesions," The Journal of Pathology, vol. 217, no. 5, pp. 716-727, 2009.

[29] A. F. West, M. O'Donnell, R. G. Charlton, D. E. Neal, and H. Y. Leung, "Correlation of vascular endothelial growth factor expression with fibroblast growth factor-8 expression and clinico-pathologic parameters in human prostate cancer," British Journal of Cancer, vol. 85, no. 4, pp. 576-583, 2001.

[30] Y. H. Gao, L. J. Yang, and A. Yamaguchi, "Immunohistochemical demonstration of bone morphogenetic protein in odontogenic tumors," Journal of Oral Pathology \& Medicine, vol. 26, no. 6, pp. 273-277, 1997.

[31] E. B. das Neves Silva Maia Pimentel, Avaliaçãoimuno-histoquímica das proteinas BMP-4, FGF-8 e Sindecan-1 emtumoresodontogênicos, 108f. Tese (DoutoradoemPatologia Oral) Centro de Ciências da Saúde, Universidade Federal do Rio Grande do Norte, Natal, 2015, Available from: http:// repositorio.ufrn.br/handle/123456789/19912. 


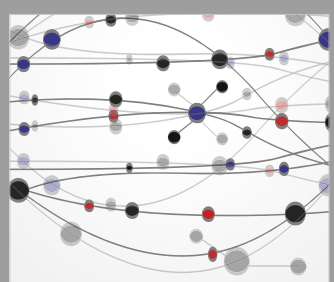

The Scientific World Journal
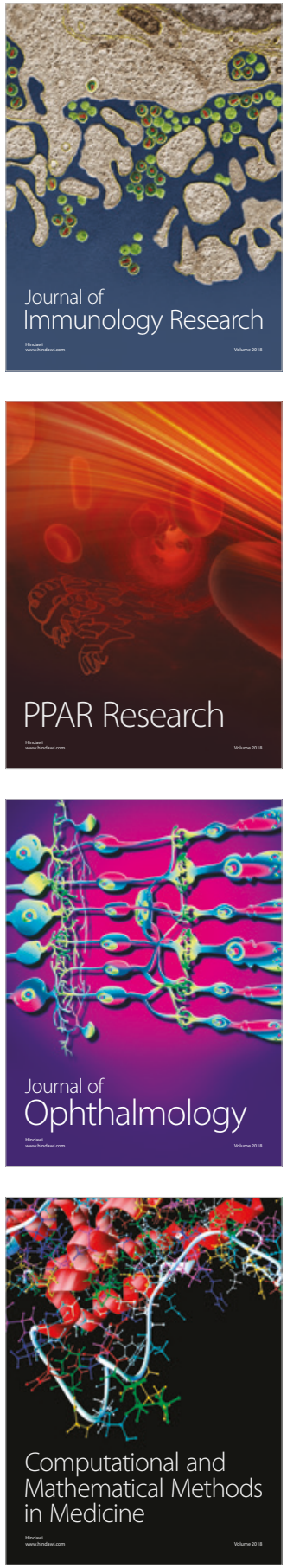

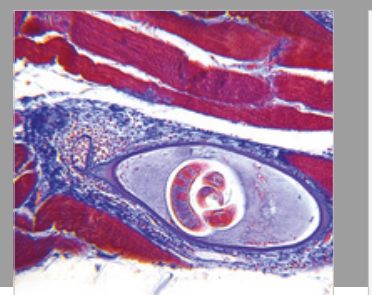

Gastroenterology Research and Practice

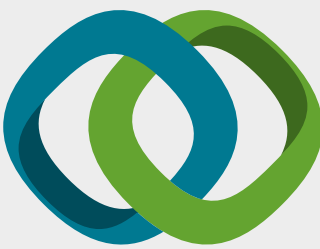

\section{Hindawi}

Submit your manuscripts at

www.hindawi.com
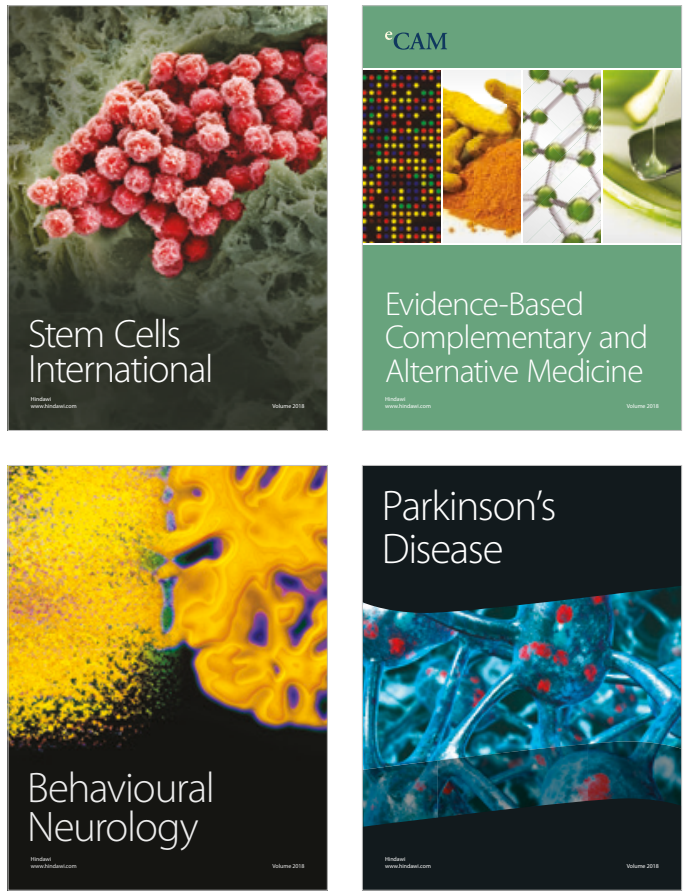

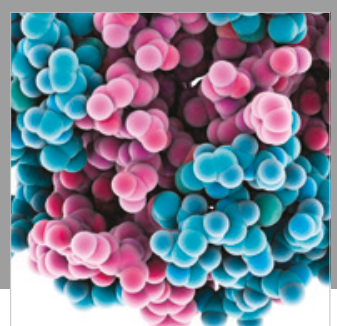

ournal of

Diabetes Research

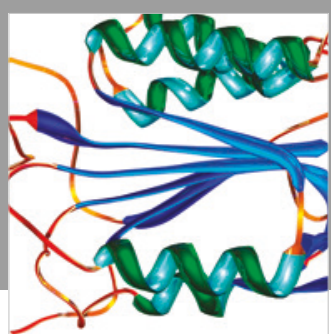

Disease Markers
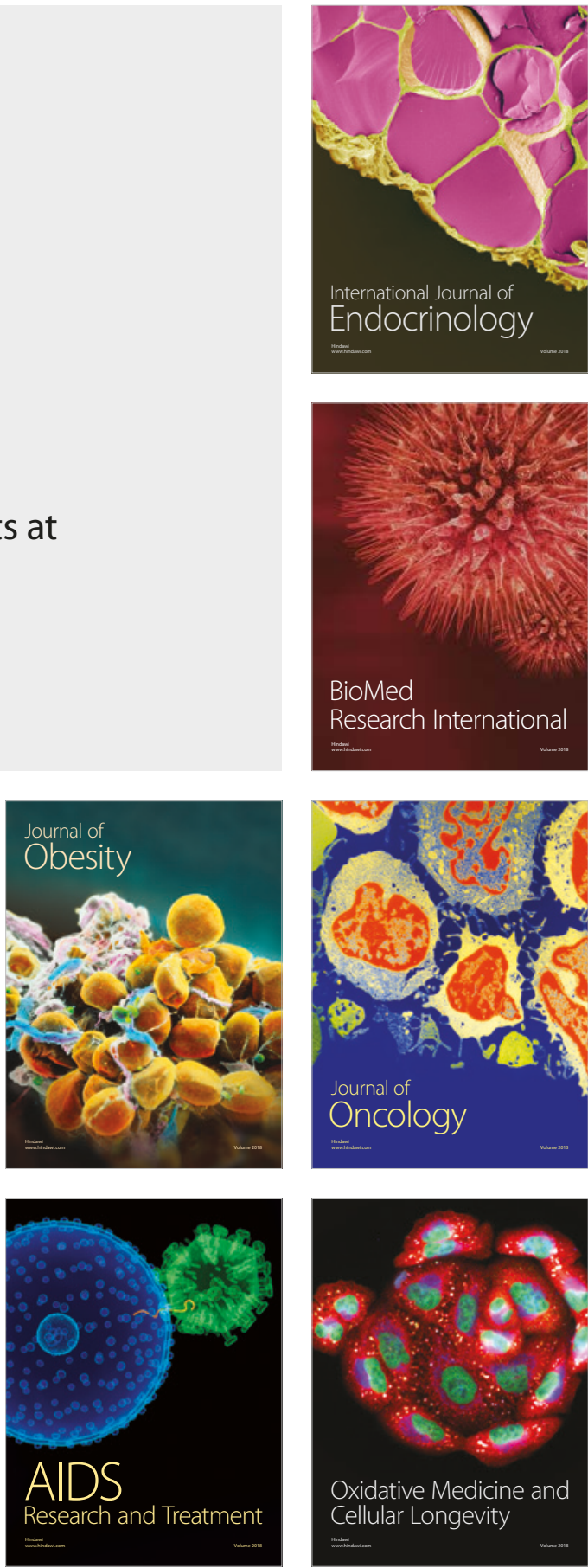